\title{
New Results of Intuitionistic Fuzzy Soft Set
}

\author{
Said Broumi \\ Faculty of Arts and Humanities, Hay El Baraka Ben M'sik Casablanca B.P. 7951, \\ Hassan II University Mohammedia-Casablanca, Morocco \\ broumisaid78@gmail.com \\ Florentin Smarandache \\ Department of Mathematics, University of New Mexico, 705 Gurley Avenue, Gallup, NM 87301, USA \\ fsmarandache@gmail.com \\ Mamoni Dhar \\ Department of Mathematics, Science College, Kokrajhar-783370, Assam, India \\ mamonidhar@gmail.com \\ Pinaki Majumdar \\ Departement of Mathematics,M.U.C Women's College, Burdwan, West-Bengal, India PIN-713104 \\ pmajumdar2@rediffmail.com
}

\begin{abstract}
In this paper, three new operations are introduced on intuitionistic fuzzy soft sets . They are based on concentration, dilatation and normalization of intuitionistic fuzzy sets. Some examples of these operations were given and a few important properties were also studied.
\end{abstract}

Index Terms - Soft Set, Intuitionistic Fuzzy Soft Set, Concentration, Dilatation, Normalization.

\section{INTRODUCTION}

The concept of the intuitionistic fuzzy (IFS, for short) was introduced in 1983 by K. Aanassov [1] as an extension of Zadeh's fuzzy set. All operations, defined over fuzzy sets were transformed for the case the IFS case. This concept is capable of capturing the information that includes some degree of hesitation and applicable in various fields of research. For example, in decision making problems, particularly in the case of medical diagnosis, sales analysis, new product marketing, financial services, etc. Atanassov et.al [2,3] have widely applied theory of intuitionistic sets in logic programming, Szmidt and Kacprzyk [4] in group decision making, De et al [5] in medical diagnosis etc. Therefore in various engineering application, intuitionistic fuzzy sets techniques have been more popular than fuzzy sets techniques in recent years. Another important concept that addresses uncertain information is the soft set theory originated by Molodtsov [6]. This concept is free from the parameterization inadequacy syndrome of fuzzy set theory, rough set theory, probability theory. Molodtsov has successfully applied the soft set theory in many different fields such as smoothness of functions, game theory, operations research, Riemann integration, Perron integration, and probability. In recent years, soft set theory has been received much attention since its appearance. There are many papers devoted to fuzzify the concept of soft set theory which leads to a series of mathematical models such as fuzzy soft set [7,8,9,10,11], generalized fuzzy soft set $[12,13]$, possibility fuzzy soft set [14] and so on. Thereafter, P.K.Maji and his coauthor [15] introduced the notion of intuitionistic fuzzy soft set which is based on a combination of the intuitionistic fuzzy sets and soft set models and they studied the properties of intuitionistic fuzzy soft set. Then, a lot of extensions of intuitionistic fuzzy soft have appeared such as generalized intuitionistic fuzzy soft set [16], possibility intuitionistic fuzzy soft set [17] etc.

In this paper our aim is to extend the two operations defined by Wang et al. [18] on intuitionistic fuzzy set to the case of intuitionistic fuzzy soft sets, then we define the concept of normalization of intuitionistic fuzzy soft sets and we study some of their basic properties.

This paper is arranged in the following manner .In section 2, some definitions and notions about soft set, fuzzy soft set, intuitionistic fuzzy soft set and several properties of them are presented. In section 3, we discuss the normalization intuitionistic fuzzy soft sets. In section 4, we conclude the paper.

\section{PRELIMINARIES}

In this section, some definitions and notions about soft sets and intutionistic fuzzy soft set are given. These will be useful in later sections.

Let $\mathrm{U}$ be an initial universe, and $\mathrm{E}$ be the set of all possible parameters under consideration with respect to $U$. The set of all subsets of $U$, i.e. the power set of $U$ is denoted by $\mathrm{P}(\mathrm{U})$ and the set of all intuitionistic fuzzy subsets of $U$ is denoted by $\mathrm{IF}^{\mathrm{U}}$. Let $\mathrm{A}$ be a subset of $E$. 


\subsection{Definition}

A pair $(F, A)$ is called a soft set over $U$, where $F$ is a mapping given by $\mathrm{F}: \mathrm{A} \rightarrow \mathrm{P}(\mathrm{U})$.

In other words, a soft set over $U$ is a parameterized family of subsets of the universe U. For $\varepsilon \in A, F(\varepsilon)$ may be considered as the set of $\varepsilon$-approximate elements of the soft set (F, A).

\subsection{Definition}

Let $\mathrm{U}$ be an initial universe set and $\mathrm{E}$ be the set of parameters. Let $\mathrm{IF}^{\mathrm{U}}$ denote the collection of all intuitionistic fuzzy subsets of $\mathrm{U}$. Let. $\mathrm{A} \subseteq \mathrm{E}$ pair $(\mathrm{F}, \mathrm{A})$ is called an intuitionistic fuzzy soft set over $U$ where $F$ is a mapping given by $\mathrm{F}: \mathrm{A} \rightarrow \mathrm{IF}^{\mathrm{U}}$.

\subsection{Defintion}

Let $\mathrm{F}: \mathrm{A} \rightarrow \mathrm{IF}^{\mathrm{U}}$ then $\mathrm{F}$ is a function defined as $\mathrm{F}(\varepsilon)$ $=\left\{\mathrm{X}, \boldsymbol{\mu}_{\boldsymbol{F}(\varepsilon)}(\boldsymbol{x}), \boldsymbol{v}_{\boldsymbol{F}(\varepsilon)}(\boldsymbol{x}): \boldsymbol{x} \in \boldsymbol{U}, \varepsilon \in \boldsymbol{E}\right\}$ where $\mu, v$ denote the degree of membership and degree of nonmembership respectively.

\subsection{Definition}

For two intuitionistic fuzzy soft sets $(\mathrm{F}, \mathrm{A})$ and $(\mathrm{G}, \mathrm{B})$ over a common universe $U$, we say that $(F, A)$ is an intuitionistic fuzzy soft subset of $(G, B)$ if

(1) $\mathrm{A} \subseteq \mathrm{B}$ and

(2) $\mathrm{F}(\varepsilon) \subseteq \mathrm{G}(\varepsilon)$ for all $\varepsilon \in$ A. i.e $\boldsymbol{\mu}_{\boldsymbol{F}(\varepsilon)}(\boldsymbol{x}) \leq$ $\boldsymbol{\mu}_{\boldsymbol{G}(\varepsilon)}(\boldsymbol{x}), \boldsymbol{v}_{\boldsymbol{F}(\varepsilon)}(\boldsymbol{x}) \geq \boldsymbol{v}_{\boldsymbol{G}(\varepsilon)}(\boldsymbol{x})$ for all $\varepsilon \in \mathrm{E}$ and We write $(\mathrm{F}, \mathrm{A}) \subseteq(\mathrm{G}, \mathrm{B})$.

\subsection{Definition}

Two intuitionistic fuzzy soft sets $(F, A)$ and $(G, B)$ over a common universe $U$ are said to be soft equal if $(F$, A) is a soft subset of $(G, B)$ and $(G, B)$ is a soft subset of (F, A).

\subsection{Definition}

Let $\mathrm{U}$ be an initial universe, $\mathrm{E}$ be the set of parameters, and $\mathrm{A} \subseteq \mathrm{E}$.

(a) $(F, A)$ is called a null intuitionistic fuzzy soft set (with respect to the parameter set $\mathrm{A}$ ), denoted by $\varphi_{A}$, if $\mathrm{F}(\mathrm{a})=$ $\varphi$ for all $\mathrm{a} \in \mathrm{A}$.

(b) $(\mathrm{G}, \mathrm{A})$ is called an absolute intuitionistic fuzzy soft set (with respect to the parameter set $\mathrm{A}$ ), denoted by $U_{A}$, if $\mathrm{G}(\mathrm{e})=\mathrm{U}$ for all $\mathrm{e} \in \mathrm{A}$.

\subsection{Definition}

Let $(\mathrm{F}, \mathrm{A})$ and $(\mathrm{G}, \mathrm{B})$ be two IFSSs over the same universe $U$. Then the union of $(F, A)$ and $(G, B)$ is denoted by ' $(F, A) \cup(G, B)$ ' and is defined by $(F, A) \cup$ $(\mathrm{G}, \mathrm{B})=(\mathrm{H}, \mathrm{C})$, where $\mathrm{C}=\mathrm{A} \cup \mathrm{B}$ and the truthmembership, falsity-membership of $(\mathrm{H}, \mathrm{C})$ are as follows:

$$
\begin{gathered}
H(\varepsilon) \\
=\left\{\begin{array}{c}
\left\{\left(\boldsymbol{\mu}_{F(\varepsilon)}(\boldsymbol{x}), \boldsymbol{v}_{\boldsymbol{F}(\varepsilon)}(\boldsymbol{x}): \boldsymbol{x} \in \boldsymbol{U}\right\}, \text { if } \varepsilon \in \mathrm{A}-\mathrm{B},\right. \\
\left\{\left(\boldsymbol{\mu}_{G(\varepsilon)}(\boldsymbol{x}), \boldsymbol{v}_{G(\varepsilon)}(\boldsymbol{x}): \boldsymbol{x} \in \boldsymbol{U}\right\}, \text { if } \varepsilon \in \mathrm{B}-\mathrm{A}\right. \\
\left\{\max \left(\boldsymbol{\mu}_{\boldsymbol{F}(\varepsilon)}(\boldsymbol{x}), \boldsymbol{\mu}_{G(\varepsilon)}(\boldsymbol{x})\right), \min \left(\boldsymbol{v}_{\boldsymbol{F}(\varepsilon)}(\boldsymbol{x}), \boldsymbol{v}_{G(\varepsilon)}(\boldsymbol{x})\right): \boldsymbol{x} \in \boldsymbol{U}\right\}, \\
\text { if } \varepsilon \in \mathrm{A} \cap \mathrm{B}
\end{array}\right.
\end{gathered}
$$

Where $\boldsymbol{\mu}_{\boldsymbol{H}(\varepsilon)}(\boldsymbol{x})=\max \left(\boldsymbol{\mu}_{\boldsymbol{F}(\varepsilon)}(\boldsymbol{x}), \boldsymbol{\mu}_{\boldsymbol{G}(\varepsilon)}(\boldsymbol{x})\right)$ and $\boldsymbol{v}_{\boldsymbol{H}(\varepsilon)}(\boldsymbol{x})=\min \left(\boldsymbol{v}_{\boldsymbol{F}(\varepsilon)}(\boldsymbol{x}), \boldsymbol{v}_{\boldsymbol{G}(\varepsilon)}(\boldsymbol{x})\right)$

\subsection{Definition}

Let $(F, A)$ and $(G, B)$ be two IFSSs over the same universe $U$ such that $A \cap B \neq 0$. Then the intersection of $(\mathrm{F}, \mathrm{A})$ and $(\mathrm{G}, \mathrm{B})$ is denoted by ' $(\mathrm{F}, \mathrm{A}) \cap(\mathrm{G}, \mathrm{B})$ ' and is defined by $(\mathrm{F}, \mathrm{A}) \cap(\mathrm{G}, \mathrm{B})=(\mathrm{K}, \mathrm{C})$, where $\mathrm{C}=\mathrm{A}$ $\cap B$ and the truth-membership, falsity-membership of ( $\mathrm{K}, \mathrm{C})$ are related to those of $(\mathrm{F}, \mathrm{A})$ and $(\mathrm{G}, \mathrm{B})$ by:

$$
\begin{aligned}
& \boldsymbol{K}(\varepsilon) \\
& =\left\{\begin{array}{c}
\left\{\left(\boldsymbol{\mu}_{F(\varepsilon)}(\boldsymbol{x}), \boldsymbol{v}_{\boldsymbol{F}(\varepsilon)}(\boldsymbol{x}): \boldsymbol{x} \in \boldsymbol{U}\right\}, \text { if } \varepsilon \in \mathrm{A}-\mathrm{B},\right. \\
\left\{\left(\boldsymbol{\mu}_{G(\varepsilon)}(\boldsymbol{x}), \boldsymbol{v}_{G(\varepsilon)}(\boldsymbol{x}): \boldsymbol{x} \in \boldsymbol{U}\right\}, \text { if } \varepsilon \in \mathrm{B}-\mathrm{A}\right. \\
\left\{\min \left(\boldsymbol{\mu}_{\boldsymbol{F}(\varepsilon)}(\boldsymbol{x}), \boldsymbol{\mu}_{G(\varepsilon)}(\boldsymbol{x})\right), \max \left(\boldsymbol{v}_{\boldsymbol{F}(\varepsilon)}(\boldsymbol{x}), \boldsymbol{v}_{\boldsymbol{G}(\varepsilon)}(\boldsymbol{x})\right): \boldsymbol{x} \in \boldsymbol{U}\right\}, \\
\text { if } \varepsilon \in \mathrm{A} \cap \mathrm{B}
\end{array}\right.
\end{aligned}
$$

\section{CONCENTRATION OF INTUITIONISTIC FUZZY SOFT SET}

\subsection{Definition}

The concentration of an intuitionistic fuzzy soft set $(\mathrm{F}$, A) of universe $\mathrm{U}$, denoted by $\mathbf{C O N}(\mathrm{F}, \mathrm{A})$, and is defined as a unary operation on $\mathrm{IF}^{\mathrm{U}}$ :

$$
\text { Con: } \mathrm{IF}^{\mathrm{U}} \rightarrow \mathrm{IF}^{\mathrm{U}}
$$

$\operatorname{Con}(\mathrm{F}, \mathrm{A})=$

$\left\{\mathrm{Con}\{\mathrm{F}(\varepsilon)\}=\left\{<\mathrm{X}, \boldsymbol{\mu}_{\boldsymbol{F}(\varepsilon)}^{2}(x), 1-\left(1-\boldsymbol{v}_{(\boldsymbol{F}(\varepsilon))}(\boldsymbol{x})\right)^{2}>\mid \boldsymbol{x}\right.\right.$ $\in \mathrm{U}$ and $\varepsilon \in \mathrm{A}\}$. where

From $0 \leq \mu_{F(\varepsilon)}(x), v_{F(\varepsilon)}(x) \leq 1$

and $\mu_{F(\varepsilon)}(x)+v_{F(\varepsilon)}(x) \leq 1$,

we obtain $0 \leq \mu_{F(\varepsilon)}^{2}(x) \leq \mu_{F(\varepsilon)}(x)$

$1 \geq 1-\left(1-\boldsymbol{v}_{(\boldsymbol{F}(\varepsilon))}(\boldsymbol{x})\right)^{2} \geq v_{F(\varepsilon)}(x)$

and $\operatorname{Con}(\mathrm{F}, \mathrm{A}) \in \mathrm{IF}^{\mathrm{U}}$, i.e $\operatorname{Con}(\mathrm{F}, \mathrm{A}) \subseteq(\mathrm{F}, \mathrm{A})$ this means that concentration of a intuitionistic fuzzy soft set leads to a reduction of the degrees of membership.

In the following theorem, The operator "Con "reveals nice distributive properties with respect to intuitionistic union and intersection. 


\subsection{Therorem}

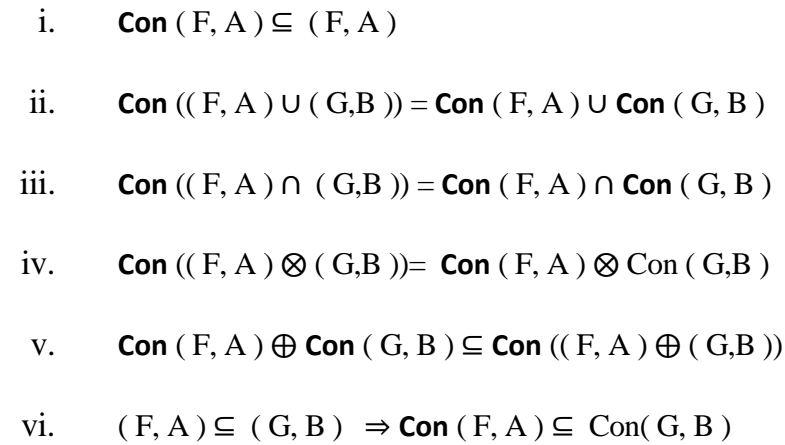

Proof, we prove only (v), i.e

$$
\begin{aligned}
& \mu_{F(\varepsilon)}^{2}(x)+\mu_{G(\varepsilon)}^{2}(x)-\mu_{F(\varepsilon)}^{2}(x) \mu_{G(\varepsilon)}^{2}(x) \leq\left(\mu_{F(\varepsilon)}(x)+\right. \\
& \left.\mu_{G(\varepsilon)}(x)-\mu_{F(\varepsilon)}(x) \mu_{G(\varepsilon)}(x)\right)^{2}, \\
& \left(1-\left(1-v_{F(\varepsilon)}(x)\right)^{2}\right) \cdot\left(1-\left(1-v_{G(\varepsilon)}(x)\right)^{2}\right) \geq 1- \\
& \left(1-v_{F(\varepsilon)}(x) \cdot v_{G(\varepsilon)}(x)\right)^{2} \text { or, putting } \\
& \mathrm{a}=\mu_{F(\varepsilon)}(x), \mathrm{b}=\mu_{G(\varepsilon)}(x), \mathrm{c}=v_{F(\varepsilon)}(x), \mathrm{d}=v_{G(\varepsilon)}(x) \\
& a^{2}+b^{2}-a^{2} b^{2} \leq(a+b-a b)^{2}, \\
& \quad\left(1-(1-c)^{2}\right) \cdot\left(1-(1-d)^{2}\right) \geq 1-(1-c \cdot d)^{2}
\end{aligned}
$$

The last inequality follows from $0 \leq \mathrm{a}, \mathrm{b}, \mathrm{c}, \mathrm{d} \leq 1$.

\section{Example}

Let $\mathrm{U}=\{\mathrm{a}, \mathrm{b}, \mathrm{c}\}$ and $\mathrm{E}=\left\{e_{1}, e_{2}, e_{3}, e_{4}\right\}, \mathrm{A}=\left\{e_{1}, e_{2}, e_{4}\right\}$ $\subseteq \mathrm{E}, \mathrm{B}=\left\{e_{1}, e_{2}, e_{3}\right\} \subseteq \mathrm{E}$

$(\mathbf{F}, \mathbf{A})=\left\{\mathbf{F}\left(\boldsymbol{e}_{\mathbf{1}}\right)=\{((\mathrm{a}, 0.5,0.1),(\mathrm{b}, 0.1,0.8),(\mathrm{c}, 0.2\right.$, $0.5)\}, \mathbf{F}\left(\boldsymbol{e}_{2}\right)=\{((\mathrm{a}, 0.7,0.1),(\mathrm{b}, 0,0.8),(\mathrm{c}, 0.3,0.5)\}$, $\mathbf{F}\left(\boldsymbol{e}_{4}\right)=\{((\mathrm{a}, 0.6,0.3),(\mathrm{b}, 0.1,0.7),(\mathrm{c}, 0.9,0.1)\}\}$

$(\mathbf{G}, \mathbf{B})=\left\{\mathbf{G}\left(\boldsymbol{e}_{1}\right)=\{((\mathrm{a}, 0.2,0.6),(\mathrm{b}, 0.7,0.1),(\mathrm{c}, 0.8\right.$, $0.1)\}, \mathbf{G}\left(\boldsymbol{e}_{2}\right)=\{((\mathrm{a}, 0.4,0.1),(\mathrm{b}, 0.5,0.3),(\mathrm{c}, 0.4,0.5)\}$, $\mathbf{G}\left(\boldsymbol{e}_{3}\right)=\{((\mathrm{a}, 0,0.6),(\mathrm{b}, 0,0.8),(\mathrm{c}, 0.1,0.5)\}\}$

Con $(\mathrm{F}, \mathrm{A})=\left\{\operatorname{con}\left(\mathbf{F}\left(\boldsymbol{e}_{1}\right)\right)=\{(\mathrm{a}, 0.25,0.19),(\mathrm{b}, 0.01\right.$, 0.96), (c, 0.04, 0.75) $\}, \operatorname{con}\left(\mathbf{F}\left(\boldsymbol{e}_{2}\right)\right)=\{(\mathrm{a}, 0.49,0.19),(\mathrm{b}, 0$, $0.96),(\mathrm{c}, 0.09,0.75)\}, \boldsymbol{c o n}\left(\mathbf{F}\left(\boldsymbol{e}_{4}\right)\right)=\{(\mathrm{a}, 0.36,0.51),(\mathrm{b}$, $0.01,0.91),(\mathrm{c}, 0.81,0.19)\}$

Con $(\mathrm{G}, \mathrm{B})=\left\{\operatorname{con}\left(\mathbf{G}\left(\boldsymbol{e}_{\mathbf{1}}\right)\right)=\{(\mathrm{a}, 0.04,0.84),(\mathrm{b}, 0.49\right.$, $0.19),(c, 0.64,0.75)\}$,

$\operatorname{con}\left(\mathbf{G}\left(\boldsymbol{e}_{2}\right)\right)=\{(\mathrm{a}, 0.16,0.19),(\mathrm{b}, 0.25,0.51),(\mathrm{c}, 0.16$, $0.51)\}, \operatorname{con}\left(\mathbf{G}\left(\boldsymbol{e}_{3}\right)\right)=\{(\mathrm{a}, 0,0.84),(\mathrm{b}, 0,0.96),(\mathrm{c}, 0.01$, $0.75)\}$

$(\mathrm{F}, \mathrm{A}) \cap(\mathrm{G}, \mathrm{B})=(\mathrm{H}, \mathrm{C})=\left\{\mathbf{H}\left(\boldsymbol{e}_{\mathbf{1}}\right)=\{(\mathrm{a}, 0.2,0.6),(\mathrm{b}, 0.1\right.$, $0.8),(\mathrm{c}, 0.2,0.5)\}, \mathbf{H}\left(\boldsymbol{e}_{2}\right)=\{(\mathrm{a}, 0.4,0.1),(\mathrm{b}, 0,0.8),(\mathrm{c}$, $0.3,0.5)\}\}$
Con $((\mathrm{F}, \mathrm{A}) \cap(\mathrm{G}, \mathrm{B}))=\left\{\operatorname{con} \mathbf{H}\left(\boldsymbol{e}_{\mathbf{1}}\right)=\{(\mathrm{a}, 0.04,0.84)\right.$, $(\mathrm{b}, 0.01,0.96),(\mathrm{c}, 0.04,0.75)\}, \operatorname{con} \mathbf{H}\left(\boldsymbol{e}_{2}\right)=\{(\mathrm{a}, 0.16$, $0.19),(\mathrm{b}, 0,0.96),(\mathrm{c}, 0.09,0.75)\}\}$

Con $(\mathrm{F}, \mathrm{A}) \cap \operatorname{Con}(\mathrm{G}, \mathrm{B})=(\mathrm{K}, \mathrm{C})=\left\{\operatorname{con} \mathbf{K}\left(\boldsymbol{e}_{\mathbf{1}}\right)=\{(\mathrm{a}\right.$, $0.04,0.84),(b, 0.01,0.96),(c, 0.04,0.75)\}, \operatorname{conK}\left(e_{2}\right)$ $=\{(\mathrm{a}, 0.16,0.19),(\mathrm{b}, 0,0.96),(\mathrm{c}, 0.09,0.75)\}\}$.

\section{Then}

$\operatorname{Con}(($ F, A $) \cap($ G,B $))=\operatorname{Con}(F, A) \cap \operatorname{Con}($ G, B $)$

\section{DILATATION OF INTUITIONISTIC FUZZY SOFT SET}

\subsection{Definition}

The dilatation of an intuitionistic fuzzy soft set $(\mathrm{F}, \mathrm{A})$ of universe U, denoted by DIL (F, A ), and is defined as a unary operation on $\mathrm{IF}^{\mathrm{U}}$ :

$$
\text { DIL: } \mathrm{IF}^{\mathrm{U}} \rightarrow \mathrm{IF}^{\mathrm{U}}
$$

$(\mathrm{F}, \mathrm{A})=\left\{\left\langle\mathrm{x}, \mu_{F(\varepsilon)}(x), v_{F(\varepsilon)}(x)>\right| x \in \mathrm{U}\right.$ and $\left.\varepsilon \in \mathrm{A}\right\}$.

$\operatorname{DIL}(\mathrm{F}, \mathrm{A})=\{$ DIL $\{\mathrm{F}(\varepsilon)\}=$

$\left\{<\mathrm{X}, \boldsymbol{\mu}_{\boldsymbol{F}(\varepsilon)}^{\frac{1}{2}}(x), 1-\left(1-\boldsymbol{v}_{(\boldsymbol{F}(\varepsilon))}(\boldsymbol{x})\right)^{\frac{1}{2}}>\mid x \in \mathrm{U}\right.$ and $\left.\varepsilon \in \mathrm{A}\right\}$.

where

From $0 \leq \mu_{F(\varepsilon)}(x), v_{F(\varepsilon)}(x) \leq 1$,

and $\mu_{F(\varepsilon)}(x)+v_{F(\varepsilon)}(x) \leq 1$,

we obtain $0 \leq \mu_{F(\varepsilon)}(x) \leq \mu_{F(\varepsilon)}^{\frac{1}{2}}(x)$

$$
0 \leq\left(1-\boldsymbol{v}_{(\boldsymbol{F}(\varepsilon))}(\boldsymbol{x})\right)^{\frac{1}{2}} \leq v_{F(\varepsilon)}(x)
$$

and $\operatorname{DIL}(\mathrm{F}, \mathrm{A}) \in \mathrm{IF}^{\mathrm{U}}$, i.e ( F, A $) \subseteq \operatorname{DIL}(\mathrm{F}, \mathrm{A})$ this means that dilatation of an intuitionistic fuzzy soft set leads to an increase of the degrees of membership.

\subsection{Theorem}

i. $(\mathrm{F}, \mathrm{A}) \subseteq \operatorname{DIL}(\mathrm{F}, \mathrm{A})$

ii. $\quad$ DIL $(($ F, A $) \cup($ G, B $))=$ DIL ( F, A $) \cup$ DIL ( G, B $)$

iii. DIL $(($ F, A $) \cap($ G, B $))=\operatorname{DIL}(F, A) \cap \operatorname{DIL}($ G, B $)$

iv. $\quad \operatorname{DIL}((\mathrm{F}, \mathrm{A}) \otimes(\mathrm{G}, \mathrm{B}))=\operatorname{DIL}(\mathrm{F}, \mathrm{A}) \otimes \operatorname{DIL}(\mathrm{G}, \mathrm{B})$

v. $\quad$ DIL $(F, A) \oplus$ DIL $(\mathrm{G}, \mathrm{B}) \subseteq$ DIL $((\mathrm{F}, \mathrm{A}) \oplus(\mathrm{G}, \mathrm{B}))$

vi. $\quad$ CON $($ DIL $(\mathrm{F}, \mathrm{A}))=(\mathrm{F}, \mathrm{A})$

vii. $\quad$ DIL $($ CON $(F, A)=(F, A)$

viii. $\quad(\mathrm{F}, \mathrm{A}) \subseteq(\mathrm{G}, \mathrm{B}) \Rightarrow \mathrm{DIL}(\mathrm{F}, \mathrm{A}) \subseteq \operatorname{DIL}(\mathrm{G}, \mathrm{B})$ 
Proof .we prove only (v), i.e

$$
\begin{aligned}
& \mu_{F(\varepsilon)}^{\frac{1}{2}}(x)+\mu_{G(\varepsilon)}^{\frac{1}{2}}(x)-\mu_{F(\varepsilon)}^{\frac{1}{2}}(x) \mu_{G(\varepsilon)}^{\frac{1}{2}}(x) \geq\left(\mu_{F(\varepsilon)}(x)+\right. \\
& \left.\mu_{G(\varepsilon)}(x)-\mu_{F(\varepsilon)}(x) \mu_{G(\varepsilon)}(x)\right)^{\frac{1}{2}}, \\
& \left(1-\left(1-v_{F(\varepsilon)}(x)\right)^{\frac{1}{2}}\right) \cdot\left(1-\left(1-v_{G(\varepsilon)}(x)\right)^{\frac{1}{2}}\right) \leq 1- \\
& \left(1-v_{F(\varepsilon)}(x) \cdot v_{G(\varepsilon)}(x)\right)^{\frac{1}{2}} \text { or, putting } \\
& \mathrm{a}=\mu_{F(\varepsilon)}(x), \mathrm{b}=\mu_{G(\varepsilon)}(x), \mathrm{c}=v_{F(\varepsilon)}(x), \mathrm{d}=v_{G(\varepsilon)}(x) \\
& a^{\frac{1}{2}}+b^{\frac{1}{2}}-a^{\frac{1}{2}} b^{\frac{1}{2}} \leq(a+b-a b)^{\frac{1}{2}}, \\
& \quad\left(1-\quad(1-c)^{\frac{1}{2}}\right) \cdot\left(1-(1-d)^{\frac{1}{2}}\right) \leq 1-(1-c \cdot d)^{\frac{1}{2}}, \text { or } \\
& \quad \text { equivalently }: \mathrm{a}+\mathrm{b}-\mathrm{a} \mathrm{b} \leq 1, \sqrt{1-c d} \leq 1 .
\end{aligned}
$$

The last inequality follows from $0 \leq \mathrm{a}, \mathrm{b}, \mathrm{c}, \mathrm{d} \leq 1$.

\section{Example}

Let $\mathrm{U}=\{\mathrm{a}, \mathrm{b}, \mathrm{c}\}$ and $\mathrm{E}=\left\{e_{1}, e_{2}, e_{3}, e_{4}\right\}, \mathrm{A}=\left\{e_{1}, e_{2}, e_{4}\right\}$ $\subseteq \mathrm{E}, \mathrm{B}=\left\{e_{1}, e_{2}, e_{3}\right\} \subseteq \mathrm{E}$

$(\mathrm{F}, \mathrm{A})=\left\{\mathbf{F}\left(\boldsymbol{e}_{1}\right)=\{((\mathrm{a}, 0.5,0.1),(\mathrm{b}, 0.1,0.8),(\mathrm{c}, 0.2\right.$, $0.5)\}, \mathbf{F}\left(\boldsymbol{e}_{2}\right)=\{((\mathrm{a}, 0.7,0.1),(\mathrm{b}, 0,0.8),(\mathrm{c}, 0.3,0.5)\}$, $\mathbf{F}\left(\boldsymbol{e}_{4}\right)=\{((\mathrm{a}, 0.6,0.3),(\mathrm{b}, 0.1,0.7),(\mathrm{c}, 0.9,0.1)\}\}$ and

$(\mathrm{G}, \mathrm{B})=\left\{\mathbf{G}\left(\boldsymbol{e}_{\mathbf{1}}\right)=\{((\mathrm{a}, 0.2,0.6),(\mathrm{b}, 0.7,0.1),(\mathrm{c}, 0.8\right.$, $0.1)\}, \mathbf{G}\left(\boldsymbol{e}_{2}\right)=\{((\mathrm{a}, 0.4,0.1),(\mathrm{b}, 0.5,0.3),(\mathrm{c}, 0.4,0.5)\}$, $\mathbf{G}\left(\boldsymbol{e}_{3}\right)=\{((\mathrm{a}, 0,0.6),(\mathrm{b}, 0,0.8),(\mathrm{c}, 0.1,0.5)\}\}$

$\operatorname{DIL}(\mathrm{F}, \mathrm{A})=\left\{\operatorname{DIL}\left(\mathrm{F}\left(e_{1}\right)\right)=\{(\mathrm{a}, 0.70,0.05),(\mathrm{b}, 0.31\right.$, $0.55),(\mathrm{c}, 0.44,0.29)\}, \operatorname{DIL}\left(\mathrm{F}\left(e_{2}\right)\right)=\{(\mathrm{a}, 0.83,0.05),(\mathrm{b}$, $0,0.55),(\mathrm{c}, 0.54,0.29)\}, \operatorname{DIL}\left(\mathrm{F}\left(e_{4}\right)\right)=\{(\mathrm{a}, 0.77,0.05)$, (b, 0.31, 0.45), (c, 0.94, 0.05) $\}$ and

DIL $(\mathrm{G}, \mathrm{B})=\left\{\right.$ DIL $\left(\mathrm{G}\left(e_{1}\right)\right)=\{(\mathrm{a}, 0.44,0.36),(\mathrm{b}, 0.83$, $0.05),(\mathrm{c}, 0.89,0.05)\}$,

$\operatorname{DIL}\left(\mathrm{G}\left(e_{2}\right)\right)=\{(\mathrm{a}, 0.63,0.05),(\mathrm{b}, 0.70,0.05),(\mathrm{c}, 0.63$, $0.29)\}, \operatorname{DIL}\left(\mathrm{G}\left(e_{3}\right)\right)=\{(\mathrm{a}, 0,0.36),(\mathrm{b}, 0,0.55),(\mathrm{c}, 0.31$, $0.29)\}$

$(\mathrm{F}, \mathrm{A}) \cap(\mathrm{G}, \mathrm{B})=(\mathrm{H}, \mathrm{C})=\left\{\mathbf{H}\left(\boldsymbol{e}_{\mathbf{1}}\right)=\{(\mathrm{a}, 0.2,0.6),(\mathrm{b}, 0.1\right.$, $0.8),(\mathrm{c}, 0.2,0.5)\}, \mathbf{H}\left(\boldsymbol{e}_{2}\right)=\{(\mathrm{a}, 0.4,0.1),(\mathrm{b}, 0,0.8),(\mathrm{c}$, $0.3,0.5)\}\}$

DIL $((\mathrm{F}, \mathrm{A}) \cap(\mathrm{G}, \mathrm{B}))=\left\{\operatorname{DILH}\left(\boldsymbol{e}_{\mathbf{1}}\right)=\{(\mathrm{a}, 0.44,0.36),(\mathrm{b}\right.$, $0.31,0.55),(\mathrm{c}, 0.44,0.29)\}, \operatorname{DILH}\left(\boldsymbol{e}_{2}\right)=\{(\mathrm{a}, 0.63,0.05)$, (b, 0, 0.55), (c, 0.54, 0. 29)\}\}

DIL ( F, A $) \cap$ DIL $(\mathrm{G}, \mathrm{B})=(\mathrm{K}, \mathrm{C})=\left\{\right.$ DIL K $\left(\boldsymbol{e}_{\mathbf{1}}\right)=\{(\mathrm{a}$, 0.04, 0.84), (b, 0.01, 0.96), (c, 0.04, 0. 75) \}, DIL K $\left(\boldsymbol{e}_{2}\right)$ $=\{(\mathrm{a}, 0.16,0.19),(\mathrm{b}, 0,0.96),(\mathrm{c}, 0.09,0.75)\}\}$

\section{Then}

$\operatorname{DIL}((F, A) \cap(G, B))=\operatorname{DIL}(F, A) \cap \operatorname{DIL}(G, B)$

\section{NORMALIZATION OF INTUITIONISTIC FUZZY SOFT SET}

In this section, we shall introduce the normalization operation on intuitionistic fuzzy soft set.

\subsection{Definition:}

The normalization of an intuitionistic fuzzy soft set ( F, A ) of universe $U$,denoted by

NORM (F, A) is defined as:

$\operatorname{NORM}(\mathrm{F}, \mathrm{A})=\left\{\operatorname{Norm}\{\mathrm{F}(\varepsilon)\}=\left\{\left\langle\mathrm{x}, \boldsymbol{\mu}_{\text {Norm }(\boldsymbol{F}(\varepsilon))}(\boldsymbol{x})\right.\right.\right.$, $\boldsymbol{v}_{\text {Norm }(\boldsymbol{F}(\varepsilon))}(\boldsymbol{x}),>\mid \boldsymbol{x} \in \mathrm{U}$ and $\left.\varepsilon \in \mathrm{A}\right\}$. where

$\boldsymbol{\mu}_{\operatorname{Norm}(F(\varepsilon))}(\boldsymbol{x})=\frac{\mu_{F(\varepsilon)}(x)}{\sup \left(\mu_{F(\varepsilon)}(x)\right)}$ and $\boldsymbol{v}_{\operatorname{Norm}(F(\varepsilon))}(\boldsymbol{x})=$ $\frac{\boldsymbol{v}_{F(\varepsilon)}(x)-\inf \left(\boldsymbol{v}_{F(\varepsilon)}(x)\right)}{1-\inf \left(\boldsymbol{v}_{F(\varepsilon)}(x)\right)}$ and

$\operatorname{Inf}\left(\boldsymbol{v}_{\boldsymbol{F}(\varepsilon)}(\boldsymbol{x})\right) \neq \mathbf{0}$.

Example. Let there are five objects as the universal set where $U=\left\{x_{1}, x_{2}, x_{3}, x_{4}, x_{5}\right\}$ and the set of parameters as $\mathrm{E}=\{$ beautiful, moderate, wooden, muddy, cheap, costly $\}$ and Let $\mathrm{A}=$ \{beautiful, moderate, wooden $\}$. Let the attractiveness of the objects represented by the intuitionistic fuzzy soft sets $(\mathrm{F}, \mathrm{A})$ is given as

$\mathrm{F}($ beautiful $)=\left\{\mathrm{x}_{1 /(.6,4)}, \mathrm{x}_{2 /(.7, .3)}, \mathrm{x}_{3 /(.5, .5)}, \mathrm{x}_{4 /(.8, .2)}\right.$, $\left.\mathrm{X}_{5 /(.9, .1)}\right\}$,

$\mathrm{F}($ moderate $)=\left\{\mathrm{x}_{1 /(.3, .7)}, \mathrm{x}_{2 /(6, .4)}, \mathrm{x}_{3 /(.8, .2)}, \mathrm{x}_{4 /(.3, .7)}\right.$, $\left.\mathrm{X}_{5 /(1, .9)}\right\}$ and

$\mathrm{F}($ wooden $)=\left\{\mathrm{x}_{1 /(.4, .6)}, \mathrm{x}_{2 /(.6, .4)}, \mathrm{x}_{3 /(.5, .5)}, \mathrm{x}_{4 /(.2, .8)}\right.$, $\left.\mathrm{X}_{5 /(.3, .7,)}\right\}$.

Then,

$\sup \left(\boldsymbol{\mu}_{\boldsymbol{F}(\text { beautiful })}(\boldsymbol{x})\right)=0.9, \inf \left(\boldsymbol{v}_{\boldsymbol{F} \text { (beautiful) }}(\boldsymbol{x})=0.1 . \mathrm{We}\right.$ have

$\boldsymbol{\mu}_{\text {Norm }(\boldsymbol{F}(\text { beautiful }))}\left(\boldsymbol{x}_{1}\right)=\frac{0.6}{0.9}=0.66$,

$\boldsymbol{\mu}_{\text {Norm (F(beautiful)) }}\left(x_{2}\right)=\frac{0.7}{0.9}=0.77$,

$\boldsymbol{\mu}_{\operatorname{Norm}(\boldsymbol{F}(\text { beautiful }))}\left(\boldsymbol{x}_{3}\right)=\frac{0.5}{0.9}=0.55$,

$\boldsymbol{\mu}_{\text {Norm (F (beautiful) })}\left(\boldsymbol{x}_{\mathbf{4}}\right)=\frac{0.8}{0.9}=0.88$,

$\boldsymbol{\mu}_{\boldsymbol{N o r m}(\boldsymbol{F} \text { (beautiful) })}\left(\boldsymbol{x}_{\mathbf{5}}\right)=\frac{0.9}{0.9}=1$ and

$\boldsymbol{v}_{\text {Norm (F (beautiful) })}\left(\boldsymbol{x}_{1}\right)=\frac{0.3}{0.9}=0.33$,

$\boldsymbol{v}_{\text {Norm (F (beautiful) })}\left(x_{2}\right)=\frac{0.2}{0.9}=0.22$, 


$$
\begin{aligned}
& \boldsymbol{v}_{\boldsymbol{N o r m}(\boldsymbol{F}(\text { beautiful }))}\left(\boldsymbol{x}_{3}\right)=\frac{\mathbf{0 . 4}}{0.9}=0.44 \\
& \boldsymbol{v}_{\boldsymbol{N o r m}(\boldsymbol{F}(\text { beautiful }))}\left(\boldsymbol{x}_{\mathbf{4}}\right)=\frac{\mathbf{0 . 1}}{0.9}=0.11, \\
& \boldsymbol{v}_{\boldsymbol{N o r m}(\boldsymbol{F} \text { (beautiful) })}\left(\boldsymbol{x}_{\mathbf{5}}\right)=\frac{\mathbf{0 . 0}}{0.9}=0 .
\end{aligned}
$$

$\operatorname{Norm}(\mathrm{F}($ beautiful $))=\left\{\mathrm{x}_{1 /(.66, .33)}, \mathrm{x}_{2 /(.77, .22)}, \mathrm{x}_{3 /(.55, .44)}\right.$, $\left.\mathrm{X}_{4 /(.88, .11)}, \mathrm{X}_{5 /(1,0)}\right\}$.

$\sup \left(\boldsymbol{\mu}_{\boldsymbol{F}(\text { moderate })}(\boldsymbol{x})\right)=0.8, \inf \left(\boldsymbol{v}_{\boldsymbol{F} \text { (moderate })}(\boldsymbol{x})=0.2\right.$. We have

$$
\begin{aligned}
& \boldsymbol{\mu}_{\text {Norm (F(moderate }))}\left(\boldsymbol{x}_{1}\right)=\frac{0.3}{0.8}=0.375, \\
& \boldsymbol{\mu}_{\text {Norm (F(moderate }))}\left(\boldsymbol{x}_{2}\right)=\frac{0.6}{0.8}=0.75, \\
& \boldsymbol{\mu}_{\text {Norm (F(moderate }))}\left(x_{3}\right)=\frac{0.8}{0.8}=1, \\
& \boldsymbol{\mu}_{\text {Norm (F(moderate })}\left(\boldsymbol{x}_{\mathbf{4}}\right)=\frac{\mathbf{0 . 3}}{0.8}=0.375, \\
& \boldsymbol{\mu}_{\text {Norm }(\boldsymbol{F} \text { (moderate }))}\left(\boldsymbol{x}_{\mathbf{5}}\right)=\frac{\mathbf{0 . 1}}{0.8}=0.125 \text { And } \\
& \boldsymbol{v}_{\text {Norm }(\boldsymbol{F}(\text { moderate }))}\left(\boldsymbol{x}_{\mathbf{1}}\right)=\frac{\mathbf{0 . 5}}{0.8}=0.625, \\
& \boldsymbol{v}_{\text {Norm }(\boldsymbol{F}(\text { moderate }))}\left(\boldsymbol{x}_{2}\right)=\frac{\mathbf{0 . 2}}{0.8}=0.25 \\
& \boldsymbol{v}_{\text {Norm }(\boldsymbol{F} \text { (moderate })}\left(\boldsymbol{x}_{3}\right)=\frac{\mathbf{0}}{0.8}=0, \\
& \boldsymbol{v}_{\text {Norm }(\boldsymbol{F} \text { (moderate) })}\left(\boldsymbol{x}_{\mathbf{4}}\right)=\frac{0.5}{0.8}=0.625, \\
& \boldsymbol{v}_{\text {Norm }(\boldsymbol{F}(\text { moderate }))}\left(\boldsymbol{x}_{\mathbf{5}}\right)=\frac{\mathbf{0 . 7}}{0.8}=0.875 \text {. }_{0.8}
\end{aligned}
$$

$\operatorname{Norm}(F($ moderate $))=\left\{x_{1 /(.375, .625)}, x_{2 /(.75, .25)}, x_{3 /(1,0)}\right.$, $\left.\mathrm{X}_{4 /(.375, .625)}, \mathrm{X}_{5 /(0.125,0.875)}\right\}$.

$\sup \left(\boldsymbol{\mu}_{\boldsymbol{F}(\text { wooden })}(\boldsymbol{x})\right)=0.6, \inf \left(\boldsymbol{v}_{\boldsymbol{F}(\text { wooden })}(\boldsymbol{x})=0.4 \mathrm{We}\right.$ have

$$
\begin{aligned}
& \boldsymbol{\mu}_{\boldsymbol{N o r m}(\boldsymbol{F}(\text { wooden }))}\left(\boldsymbol{x}_{1}\right)=\frac{\mathbf{0 . 4}}{0.6}=0.66, \\
& \boldsymbol{\mu}_{\text {Norm (F(wooden }))}\left(\boldsymbol{x}_{2}\right)=\frac{0.6}{0.6}=1, \\
& \boldsymbol{\mu}_{\text {Norm }(\boldsymbol{F}(\text { wooden }))}\left(\boldsymbol{x}_{3}\right)=\frac{\mathbf{0 . 5}}{0.6}=0.83, \\
& \boldsymbol{\mu}_{\boldsymbol{N o r m}(\boldsymbol{F}(\text { wooden }))}\left(\boldsymbol{x}_{4}\right)=\frac{0.2}{0.6}=0.34, \\
& \boldsymbol{\mu}_{\text {Norm (F(wooden }))}\left(\boldsymbol{x}_{\mathbf{5}}\right)=\frac{0.3}{0.6}=0.5 \text { and } \\
& \boldsymbol{v}_{\text {Norm }(\boldsymbol{F}(\text { wooden }))}\left(x_{1}\right)=\frac{0.2}{0.6}=0.34,
\end{aligned}
$$

$$
\begin{aligned}
& \boldsymbol{v}_{\text {Norm }(\boldsymbol{F}(\text { wooden }))}\left(\boldsymbol{x}_{2}\right)=\frac{\mathbf{0}}{0.6}=0, \\
& \boldsymbol{v}_{\text {Norm }(\boldsymbol{F}(\text { wooden }))}\left(\boldsymbol{x}_{3}\right)=\frac{\mathbf{0 . 1}}{0.6}=0.17, \\
& \boldsymbol{v}_{\text {Norm }(\boldsymbol{F}(\text { wooden }))}\left(\boldsymbol{x}_{\mathbf{4}}\right)=\frac{\mathbf{0 . 4}}{0.6}=0.66 \text {, } \\
& \boldsymbol{v}_{\text {Norm }(\boldsymbol{F}(\text { wooden }))}\left(\boldsymbol{x}_{5}\right)=\frac{0.3}{0.6}=0.5 \text {. }
\end{aligned}
$$

$\operatorname{Norm}(\mathrm{F}($ wooden $))=\left\{\mathrm{x}_{1 /(.66, .34)}, \mathrm{x}_{2 /(1, .0)}, \mathrm{x}_{3 /(0.83,0.17)}\right.$, $\left.\mathrm{X}_{4 /(.34, .66)}, \mathrm{X}_{5 /(0.5,0.5)}\right\}$.

Then, Norm $(F, A)=\{$ Norm F (beautiful), Norm F(moderate), Norm F(wooden) $\}$

$\operatorname{Norm}(F, A)=\left\{F(\right.$ beautiful $)=\left\{x_{1 /(.66,33)}, x_{2 /(.77, .22)}\right.$, $\left.\mathrm{X}_{3 /(.55, .44)}, \mathrm{x}_{4 /(.88, .11)}, \mathrm{x}_{5 /(1,0)}\right\}, \mathrm{F}($ moderate $)=\left\{\mathrm{x}_{1 /(.375, .625)}\right.$, $\left.\mathrm{x}_{2 /(.75, .25)}, \mathrm{x}_{3 /(1,0)}, \mathrm{x}_{4 /(.375, .625)}, \mathrm{x}_{5 /(0.125,0.875)}\right\}, \mathrm{F}($ wooden $)$ $\left.=\left\{\mathrm{x}_{1 /(.66,34)}, \mathrm{x}_{2 /(1, .0)}, \mathrm{x}_{3 /(0.83,0.17)}, \mathrm{x}_{4 /(.34, .66)}, \mathrm{x}_{5 /(0.5,0.5)}\right\}\right\}$

Clearly, $\boldsymbol{\mu}_{\boldsymbol{N o r m}(\boldsymbol{F}(\varepsilon))}(\boldsymbol{x})+\boldsymbol{v}_{\boldsymbol{N o r m}(\boldsymbol{F}(\varepsilon))}(\boldsymbol{x})=1$, for i $=1,2$, $3,4,5$ which satisfies the property of intuitionistic fuzzy soft set. Therefore, Norm (F, A) is an intuitionistic fuzzy soft set.

\section{CONCLUSION}

In this paper, we have extended the two operations of intuitionistic fuzzy set introduced by Wang et al.[ 18] to the case of intuitionistic fuzzy soft sets. Then we have introduced the concept of normalization of intuitionistic fuzzy soft sets and studied several properties of these operations.

\section{ACKNOWLEDGMENTS}

The authors are highly grateful to the referees for their valuable comments and suggestions for improving the paper and finally to God who made all the things possible.

\section{REFERENCES}

[1] K.T. Atanassov," Intuitionistic Fuzzy Set”. Fuzzy Sets and Systems, vol. 20(1), pp.87-86, 1986.

[2] K.T. Atanassov and G. Gargov ," intuitionistic fuzzy logic “,C.R Academy of Bulgarian Society, Vol. 53, pp .9-12, 1990.

[3] K.T. Atanassov and G.Gargov, "intuitionistic fuzzy prolog “,Fuzzy Sets and sSystems, Vol. 4, No 3, 1993 pp .121-128.

[4] E.Szmidt and J.Kacprzyk, "Intuitionistic Fuzzy Sets in Group Decision Making", Notes on IFS 2, ,1996, pp.1114.

[5] S.K.De, R. Biswas and A.Roy, "An Application of Intuitionstic Fuzzy Set in Medical Diagnosis “, Fuzzy Sets and Systems, vol .117,2001, pp .209-213.

[6] D. A. Molodtsov, "Soft Set Theory - First Result", Computers and Mathematics with Applications, Vol. 37, 1999, pp. 19-31. 
[7] P. K. Maji, R. Biswas and A.R. Roy, "Fuzzy Soft Sets", Journal of Fuzzy Mathematics, Vol 9 , No.3, 2001, pp. 589-602.

[8] B. Ahmad and A. Kharal, "On Fuzzy Soft Sets", Hindawi Publishing Corporation, Advances in Fuzzy Systems, volume Article ID 586507, (2009), 6 pages doi: $10.1155 / 2009 / 586507$.

[9] P. K. Maji, A. R. Roy and R. Biswas, "Fuzzy Soft Sets", Journal of Fuzzy Mathematics. Vol 9, No 3, 2001, pp.589-602.

[10] T. J. Neog and D. K. Sut, "On Fuzzy Soft Complement and Related Properties", Accepted for publication in International, Journal of Energy, Information and communications (IJEIC).

[11] M. Borah, T. J. Neog and D. K. Sut," A study on some operations of fuzzy soft sets", International Journal of Modern Engineering Research (IJMER), Vol.2, No. 2, 2012, pp. 157-168.

[12] H. L. Yang, "Notes On Generalized Fuzzy Soft Sets", Journal of Mathematical Research and Exposition, Vol 31, No. 3, (2011), pp.567-570.

[13] P. Majumdar, S. K. Samanta, "Generalized Fuzzy Soft Sets", Computers and Mathematics with Applications, Vol 59, 2010, pp.1425-1432.

[14] S. Alkhazaleh, A. R. Salleh, and N. Hassan," Possibility Fuzzy Soft Set”, Advances in Decision Sciences, Vol 2011, Article ID 479756,doi:10.1155/2011/479756, 18 pages.

[15] P. K. Maji, R. Biswas, A. R. Roy, "Intuitionistic fuzzy soft sets", The journal of fuzzy mathematics Vol 9, NO3, 2001, pp.677-692.

[16] K.V .Babitha and J. J. Sunil," Generalized Intuitionistic Fuzzy Soft Sets and Its Applications "Gen. Math. Notes, ISSN 2219-7184; ICSRS Publication, (2011), Vol. 7, No. 2, 2011, pp.1-14.

[17] M.Bashir, A.R. Salleh, and S. Alkhazaleh," Possibility Intuitionistic Fuzzy Soft Set", Advances in Decision Sciences Volume 2012, 2012, Article ID 404325, 24 pages, doi:10.1155/2012/404325.

[18] W.Yang-Ping, Z.Jun,” Modifying Operations on Intuitionistic Fuzzy sets.

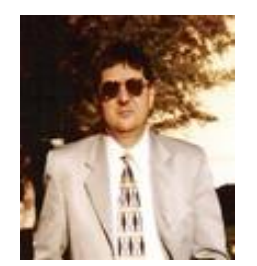

Dr. Florentin Smarandache is a Professor of Mathematics at the University of New Mexico in USA. He published over 75 books and 250 articles and notes in mathematics, physics, philosophy, psychology, rebus, literature. In mathematics his research is in number theory, non-Euclidean geometry, synthetic geometry, algebraic structures, statistics, neutrosophic logic and set (generalizations of fuzzy logic and set respectively), neutrosophic probability (generalization of classical and imprecise probability).Also, small contributions to nuclear and particle physics, information fusion, neutrosophy (a generalization of dialectics), law of sensations and stimuli, etc. He got the 2010 Telesio-Galilei Academy of Science Gold Medal, Adjunct Professor (equivalent to Doctor Honoris Causa) of Beijing Jiaotong University in 2011, and 2011 Romanian Academy Award for Technical Science (the highest in the country). Dr. W. B. Vasantha Kandasamy and Dr.Florentin Smarandache got the 2012 and 2011 New Mexico-Arizona Book Award for Algebraic Structures.

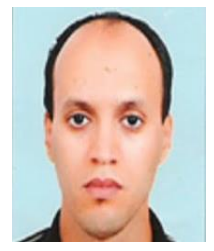

Said Broumi is an administrator in Hassan II University Mohammedia- Casablanca. He worked in University for five years. $\mathrm{He}$ received his M. Sc in Industrial Automatic from Hassan II University Ain chokCasablanca. His research concentrates on soft set theory, fuzzy theory, intuitionistic fuzzy theory, neutrosophic theory, control systems.

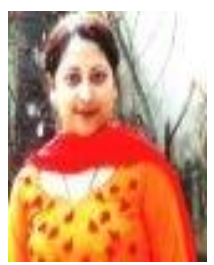

Mamoni Dhar is an Assistant Professor in the department of Mathematics, Science College, Kokrajhar, Assam, India. She received M.Sc degree from Gauhati University, M.Phil degree from Madurai Kamraj University, B.Ed from Gauhati University and PGDIM from India Gandhi National Open University. Her research interest is in Fuzzy Mathematics. She has published eighteen articles in different national and international journals.

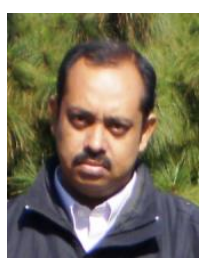

Dr. Pinaki Majumdar is an assistant professor and head of the department of Mathematics of M.U.C Women's College under University of Burdwan in INDIA. He is also a guest faculty in the department of Integrated Science Education and Research of Visva-Bharati University, INDIA. His research interest includes Soft set theory and its application, Fuzzy set theory, Fuzzy and Soft topology and Fuzzy functional analysis. He has published many research papers in reputed international journals and acted reviewer of more than a dozen of international journals. He has also completed a few projects sponsored by University Grants Commission of INDIA.

How to cite this paper: Said Broumi, Florentin Smarandache, Mamoni Dhar, Pinaki Majumdar,"New Results of Intuitionistic Fuzzy Soft Set", IJIEEB, vol.6, no.2, pp.47-52, 2014. DOI: 10.5815/ijieeb.2014.02.06 